\title{
Single molecule studies of biological processes
}

\author{
Steven Chu \\ Physics Department, Stanford University, Stanford CA 94305
}

A sampling of our recent applications of single molecule fluorescent studies to biological molecules and molecular systems is reviewed. This work represents the combined efforts of over a dozen collaborators whose contributions are cited throughout the paper.

\section{Introduction}

Virtually all knowledge of chemical and biochemical processes has been deduced from studies of a large number of molecules. This has led us to think of molecular processes as proceeding along a path of well-ordered events. However, biological processes, such as DNA replication, the synthesis of proteins by molecular machines in the cell, communication between brain cells, and the folding of proteins and RNA enzymes have complex dynamic and kinetic behavior. Many of these reactions could involve multiple pathways and transient intermediate states that would normally be invisible with conventional measurements.

In the past decades, a number of single molecule techniques have been developed and applied to biological research. The ability to record the current changes in a single ion channel revolutionized the study of ion transport in cellular membranes [1]. Single molecule studies of motor proteins [2], enzymes [3], and other proteins [4] have provided insights into the molecular mechanisms of their biological functions. Our polymer studies using DNA have fundamentally altered our thinking of polymer dynamics by showing that identical molecules placed under identical conditions take several distinct paths to a new equilibrium state [5].

Fluorescence resonance energy transfer (FRET) has been demonstrated to be an effective way to study the behavior of biomolecules [6]. Donor and acceptor dyes attached to two sites of a biological molecule can be used to measure the distance between the two dyes: donor excitation may be transferred to the acceptor molecule via near-field dipole-dipole interactions. Thus, a change in the relative fluorescence from the two dyes can be used as an indicator of a change in the separation (and orientation if the dye molecules are attached rigidly to the host molecule) of biomolecule. Since the two fluorophores are on different parts of the bio-molecule, intra-molecular motion can be measured free from the Brownian motion of the molecular center of mass.

The FRET technique has recently been extended to the single molecule domain [7]. Although interesting fluctuations were seen by observing molecules in these early studies, the precise relation of FRET changes to the molecular conformational structures was not known. Furthermore, the freely suspended molecules would drift in and out of a confocal volume so only fluctuations faster than the observation time could be studied. 
We applied single molecule fluorescence methods to study the behavior of individual immobilized biomolecules and bio-molecular systems. In our first demonstration experiment, we showed that the structural changes in individual immobilized three-helix junction RNA molecules can be unambiguously observed with FRET [8]. Since the molecules were immobilized, the change could be induced by changing the buffer conditions. In this work, we were able to flow in either the S15 protein or $\mathrm{Mg}^{2+}$ ions to induce the conformational change. Using auto and crosscorrelation methods on these immobilized individual junctions, relaxation times that spanned 5 orders of magnitude were measured [9].

Single molecule fluorescence methods were also used to study catalysis and folding of Tetrahymena ribozyme molecules [10]. We showed that dye-labeled and surface-immobilized ribozymes were functionally indistinguishable from the unmodified ribozyme free in solution, and that these methods could be used to observe RNA activity, unfolding and re-folding with the recovery of full biological activity.

By exposing the unfolded ribozyme to different salt conditions in the unfolded state, we found that the folding of the Tetrahymena ribozyme proceeds through specific pathways [11]. Additional mutagenesis experiments showed that the initial molecular contacts are formed as folding begins have profound effects on the overall folding kinetics for RNA [11].

Protein folding was also studies at a single molecule with the aid of fluorescence self-quenching [12]. Since self-quenching requires conjugation of only one type of fluorophore instead of a two-color labeling as required by FRET, the ease in labeling specific amino acids makes it very attractive for protein folding studies. In this study, we used titin as the model protein system, a multi-domain protein primarily composed of hundreds of immunoglobulin or fibronectin modules. Using an atomic force microscope assay, we showed that dye-conjugation did not perturb the unfolding of the protein and allowed it to refold to the original structure after denaturation.

\section{The hairpin ribozyme}

In this work, we show that single molecule methods can be used to develop a unified picture of the structure, the dynamics of that structure, and the biological activity of biomolecules. Previous studies of the hairpin ribozyme suggested that the enzyme follows a complex set of steps that result in the cleavage of an RNA substrate [13]. Using both FRET and a novel triple probe combination of FRET and a fluorescence quencher [14], we were able to measure the rate constants of the multiple step reaction pathway of the hairpin ribozyme as shown in Fig. 1.

In these studies, we discovered several unexpected results. (1) Four undocking rates were required to describe the observed undocking kinetics. (2) Docking occurs with the formation of several hydrogen bonds, and with additional mutation studies, we showed that these undocking rates were due to the formation of a different number of hydrogen bonds between the two loops of the hairpin ribozyme. (3) The 


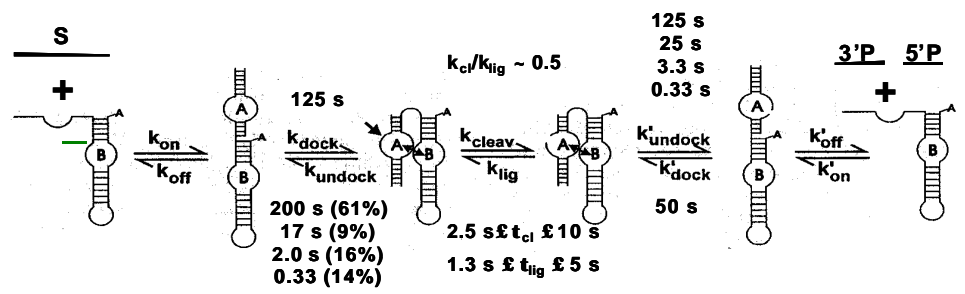

Fig. 1. The hairpin ribozyme cuts a piece of RNA into two pieces. Donor and acceptor dye molecules attached to the ends of the enzyme show changes in the FRET signal as the molecule fluctuates between the docked and undocked states. The time constants for undocking with substrate (S) attached and with products (3'P and $5^{\prime} \mathrm{P}$ ) attached were extracted from histograms of the times spent in the docked states.

undocking showed a pronounced memory effect where the time the enzyme took to undock was highly correlated with the previous undocking time as shown in Fig.2.

With the rate constants given in Fig. 1 and assuming prefect memory, we can describe the overall enzymatic activity as a set of coupled rate equations. The solution to this set of equations (with the cleavage and ligation rates and the fraction of molecules that were not biologically active as free parameters) is in excellent agreement with the data [14].

We proposed a model for the memory effect based on slow transitions between different structural configurations of loops A and B [14]. The structures of these loops in the undocked state determined by NMR [15] are different from those in the docked state determined by X-ray crystallography [16]. The model hypothesizes that loops A and B can adopt different conformations in the undocked state. If each loop has two possible conformations that favor a deeply docked and a loosely docked state, respectively, then their four combinations lead to four undocking rates, as are observed. The slow transition time between these loop conformations determines the memory time of the undocking rates. If only one of the two loops undergoes such transitions, it would require four different metastable conformations of this loop to give the four undocking rates. We plan to test this hypothesis with temperature studies and a series of mutation studies that alter the structures of loops A and B to favor certain docked states at the expense of others.

Our single molecule results demonstrate how the structural dynamics are needed to understand the catalytic function of the hairpin ribozyme. Strikingly, the hairpin ribozyme, one of the simplest RNA enzymes, shows very complex structural dynamics such as four docked states of distinct stabilities and a strong memory effect. Our observations would be difficult to obtain by ensemble methods, demonstrating the power of single molecule approaches in characterizing complex structural dynamics. 


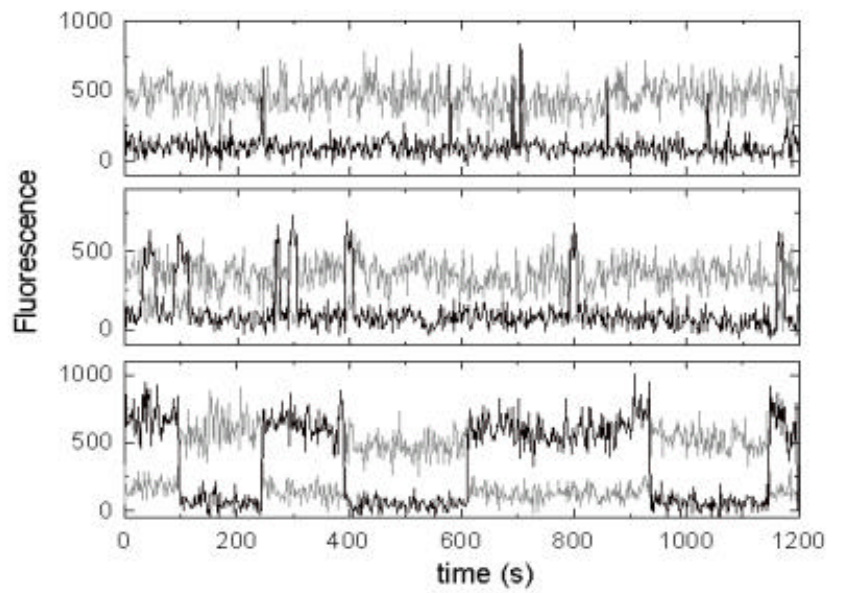

Fig. 2. Fluctuations of the donor (gray) and acceptor (black) fluorescence, showing a striking correlation of the time spent in the docked state.

\section{A study of the ribosome}

We are in the process of applying single molecule methods to study of more complex biological systems. One example is our study of a translation by the ribosome, done in collaboration with Jodi Puglisi's group in the structural Biology Department of Stanford University [17].

Translation is the mechanism of protein synthesis in which messenger RNA (mRNA) is translated into a chain of amino acids by the ribosome. Information about ribosome function and the process of translation has been largely obtained from simplified in vitro experiments, typically using purified components [18]. The enzyme consists of two subunits (50S and 30S) that assemble around the mRNA with a single initiator transfer RNA (tRNA) in the so-called P-site of the ribosome. Transfer RNA carrying specific amino acids plus the elongation factor EF-Tu and GTP are able to recognize successive three-base sequences of the mRNA by forming Watson-Crick bases pairs with the mRNA threaded through the smaller subunit of the ribosome. If the match is correct, the tRNA completes its insertion into the A-site of the ribosome by attaching to the larger subunit. A mis-match of the tRNA and mRNA prevents the completion of this insertion with a proof-reading accuracy of 5000:1. Fig. 3 shows the complete cycle of the addition of one amino acid. 

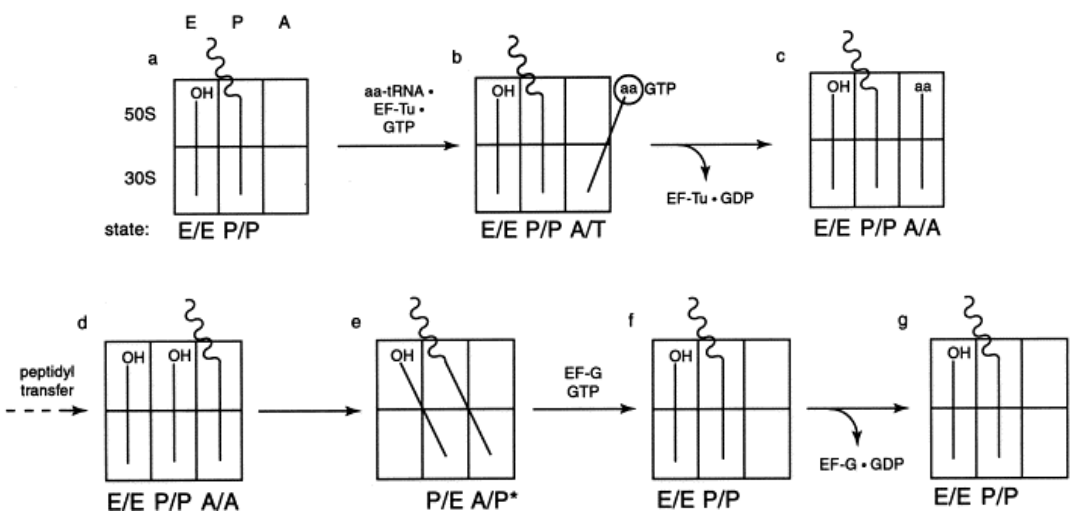

Fig. 3. The motion of tRNA through the ribosome. A tRNA carrying an amino acid (aa), elongation factor EF-Tu, and GTP enters the A-site of the $30 \mathrm{~S}$ subunit as shown in (b). With the proper base-pair matching, the tRNA completes the insertion by attaching to the Asite of the 50S subunit, ending in state (c). The amino acid chain (shown by the wiggly line) then links with aa on the A-site (d), followed by movement of both tRNA's into the hybrid state (e). An additional elongation factor EF-G is required to move the tRNA to states (f) and (g).

Recent triumphs in structural studies of the ribosome have provided atomicresolution models of the isolated ribosomal subunits and a near-atomic resolution model of the functional ribosome particle [19]. From these studies, and earlier electron microscopic reconstructions of the E.coli ribosome, we now have models for the spatial organization of all of the components of the ribosome particle that can be used to generate a physical framework to describe ribosome function. However, static views of the ribosome structure do not reveal the mechanism of translation. Information on the conformational rearrangements of the enzyme, how tRNA molecules move as they transit the ribosome, or the details of the interactions with factors required in the process of translation are not known. Our goal is to follow the motion of individual tRNA's as they move through the ribosome and correlate this motion with conformational changes of the ribosome. With these studies, we hope to develop a detailed mechanistic picture of how the ribosome functions.

To date, several important first steps towards our goal have been demonstrated. In vitro translation with immobilized ribosomes made from purified components is shown in Fig. 4. It also should be possible to decipher the individual steps the tRNA make as they enter and pass through the ribosome. By exposing dye-labeled tRNA to an immobilized ribosome, and recording the donor and acceptor fluorescence, we can watch the tRNA as it incorporates into the enzyme. Comparisons of FRET 


\section{$(4 \mathrm{a}$}

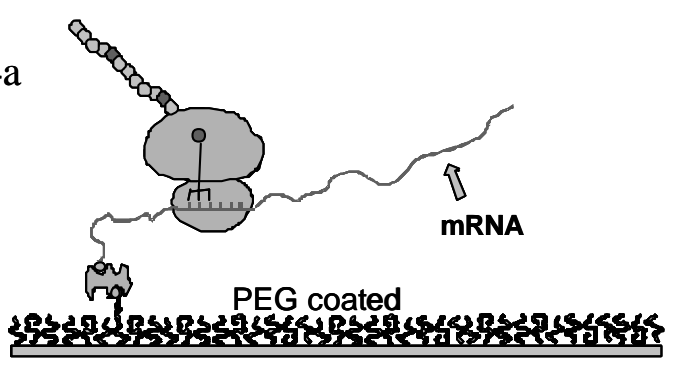

(4b)

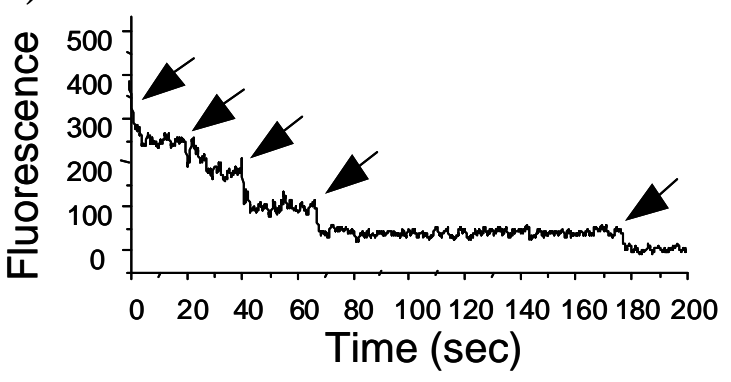

Fig. 4. (a) The mRNA - ribosome complex is immobilized on a polyethylene glycol (PEG) coated surface with biotin-strepavidinbiotin linker. When cell extract containing dye-labeled lysine tRNA is added, a message incorporating several dye-labeled amino acids is produced. (b) The dye-labeled amino acids are counted by noting the number of photobleaching events. The rate of production is comparable to our in vitro work with the ribosomes free in solution.

signals are made between aminoacylated and de-acylated tRNA (tRNA with or without an amino acid attached) in the P-site. For the deacylated sample, we see a stable FRET efficiency value $\mathrm{I}_{\text {acceptor }} /\left(\mathrm{I}_{\text {accpetor }}+\mathrm{I}_{\text {donor }}\right) \sim 0.8$ as shown in Fig. 5. If there is no amino acid on the P-site tRNA, the two tRNA's will stabilize in the P/P and A/A sites as in Fig 3c. Thus, we can identify the 0.8 FRET state with the configuration shown in Fig. 3c.

If there is an amino acid on the P-site tRNA, the reaction is presumed to continue with the formation of the peptidyl bond (Fig. 3d), and then move into the so-called "hybrid state" (Fig. 3e). Instead, we observe FRET signals that fluctuate rapidly between 0.8 and lower valued FRET states of $\sim 0.6$. Preliminary experiments suggest that the tRNA is fluctuating between the hybrid state (3e) and the pure state (3c). To test whether we are forming the hybrid state, we added puromycin, a drug that binds to the amino acid attached to a tRNA on the P/P- site. Once bound, the puromycin + amino acid releases from the tRNA and diffuses away from the ribosome. If another tRNA is in the A/A-site or if the tRNA's are in the hybrid state, the puromycin 


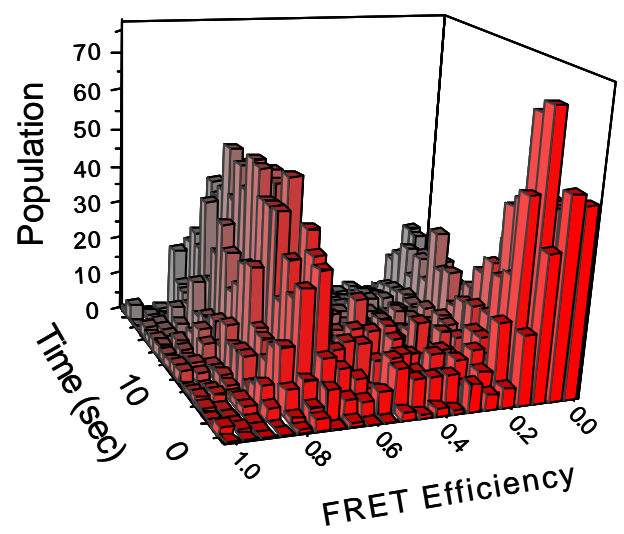

Fig. 5. A histogram of FRET efficiency vs. the time after the ternary complex is delivered to the ribosome. After $\sim 5$ seconds, a FRET peak appears at $\sim 0.8$. By illuminating the sample with lower duty cycle, we show that the peak at $\sim 0.05$ is due to the photobleaching of the acceptor dyes.

activity is greatly reduced. For these tests, we attach the dye label to the amino acid instead of the elbow of the tRNA. Upon the addition of puromycin to the ribosome with an amino-acylated tRNA in the P/P site, a rapid loss of fluorescence (release of the amino acid) is observed, as expected. With either an acylated or de-acylated tRNA in the P/P site, the addition of a ternary complex to the A-site reduces rate of puromycin release. With other experiments similar in form to the one described above, we are in the process of correlating different FRET states with different conformations of the tRNA within the ribosome. Thus, by combining structural information of the ribosome and previous biochemical experiments, we hope to construct a real-time motion picture of translation.

\section{Neural vesicle fusion}

We are also studying neural vesicle fusion in collaboration with Axel Brunger's group in the Molecular and Cellular Physiology, Neurology and Neurological Sciences Departments at Stanford University [20].

Upon the electrical firing of a nerve, voltage spikes are transmitted down the axon of the neuron. When the voltage pulse arrives at the tips of the axon, $\mathrm{Ca}^{2+}$ channels open and increase the local $\left[\mathrm{Ca}^{2+}\right]$ to increase by 2-3 orders of magnitude. This calcium increase leads to fusion of vesicles with the cell wall, and the release of neurotransmitters into the synaptic junction. The neurotransmitters diffuse rapidly across the synapse to the receptor sites of the adjacent neuron. 
The SNARE family of proteins are involved in the docking of the vesicles to the cell membrane [21]. They are also thought to be responsible for the calcium triggered fusion of the vesicle release of neurotransmitters. The fusion of synaptic vesicles with the cell membrane are characterized by their speed $(<0.1 \mathrm{msec})$, reliability and rapid adjustability. The SNARE proteins that induce vesicle fusion is strikingly similar to many other membrane fusion processes that enable compartmentalization and chemical signaling in cells.

In our experiments, artificially formed vesicles are exposed to a lipid bilayer membrane deposited on a quartz substrate and containing proteins thought to be involved in driving fusion. The SNARE proteins bind to each other to form a structure that docks the vesicle near the membrane as shown in Fig. 6. Single molecule fluorescence microscopy methods will then be used to follow the binding and dynamics of individual protein conformations.

A primary goal of this project is to discover the calcium trigger and understand the mechanism by which it operates. The temporal response of the fusion event is only known with the upper limit of about a millisecond, and one of our goals is to resolve the dynamics of this event.

Thus far, we have been successful in forming the vesicles and the deposited bilayer with the SNARE proteins. Using site-directed mutagenesis, these proteins have been expressed in forms that allow fluorescent labeling of the proteins that

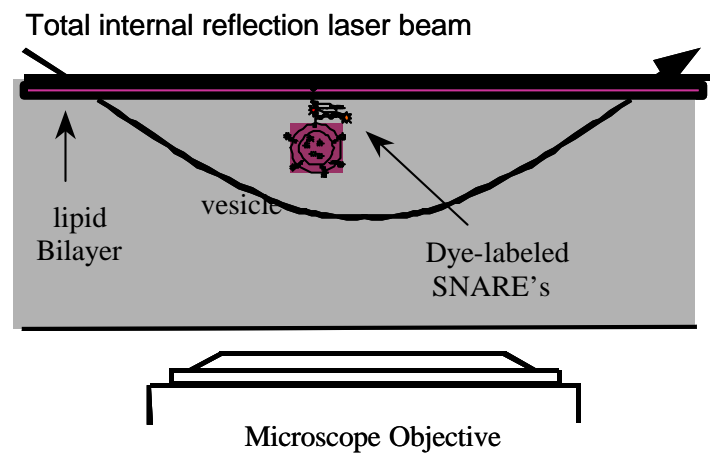

Fig. 6. Schematic of a vesicle attached to a supported bilayer via SNARE proteins. The dye molecules attached to the proteins and inserted in the vesicle is excited by a totally internally reflected laser beam that extends into the quartz-water interface.

allow the conformation of the SNARE complex to be observed with FRET. Binding between the SNAREs is observed, but much to our surprise, about $1 / 3$ of the proteins bind anti-parallel where the $\mathrm{N}$ terminus of one SNARE protein (syntaxin) is near the C-terminus of another protein (SNAP-25). This finding is in conflict with the structure of the water soluble form of protein complexes, which have been found by 
$\mathrm{X}$-ray diffraction to align in $\mathrm{N}$ to $\mathrm{N}$ terminus geometry [22]. It is conceivable that additional proteins are needed to guide the correct assembly. Alternatively, the crystallized molecules may not reflect all of the structures formed in vivo or in vitro conditions.

We have also developed a fluorescence assay in which vesicles are loaded with dye molecules at densities where they begin to self-quench each other. Upon vesicle fusion, a rapid increase in the amount of fluorescence followed by photobleaching is observed. If instead, the vesicle bursts without fusion, a rapid disappearance of fluorescence is seen as the dye molecules quickly diffuse out of the region illuminated by the totally internally reflected laser beam.

\section{Summary}

With the use of fluorescence probes attached to individual molecules, we have been able to observe rich phenomena that would have been obscured by experiments using an ensemble of many molecules. The ability to observe individual molecules reveals new phenomena such as "molecular individualism" and "molecular memory" [23]. The individual folding pathways of the Tetrahymena ribozyme can be followed in real time, the individual rates of multi-step reactions of the Hairpin ribozyme can be measured and used to understand the overall enzymatic activity of the enzyme. In that study, we were able to see how structural fluctuations play an integral role in biological function.

These successes have encouraged us to examine more complex biological systems such as translation by the ribosome and the assembly and function of the SNARE proteins implicated in neural vesicle fusion. Preliminary results suggest that single molecule methods will help us develop a more detailed, mechanistic understanding of these important biological events.

\section{References}

[1] See for example, E. Neher and B. Sakmann, Nature 260, 779 (1976).

[2] See for example, S.M. Block, L.S.B. Goldstein, and B.J. Schnapp, Nature 348. 348 (1990); Simmons, R.M., Finer, J.T., Warrick, H.M., Kralik, B., Chu, S., Spudich, J.A., Advances in Experimental Medicine and Biology, ed. H. Sugi, (1992); J.T. Finer, R.M. Simmons, and J.A. Spudich, Nature 368, 113 (1994); H. Noji, R. Yasuda, M. Yoshida, and K. Kinosita, Nature 386, 299 (1997).

[3] H.P. Lu, L.Y. Xun, and X.S. Xie, Science 282, 1877 (1998).

[4] M.S.Z. Kellermayer, S.B. Smith, H.L. Granzier and C. Bustamante, Science 276, 1112 (1997); M. Rief, M. Gautel, F. Oesterhelt, J.M. Fernandez, H. Gaub Science 276, 1109 (1997); L. Tskhovrebova, J. Trinik, J.A. Sleep and R.M. Simmons, Nature 387, 308 (1997). 
[5] T.T. Perkins, D.E. Smith and S. Chu, Science 276, 2016 (1997); D.E. Smith and S. Chu, Science 281, 1335 (1998).

[6] L. Stryer, R.P. Haugland, Proc. Natl. Acad. Sci. USA 58, 719 (1967).

[7] T. Ha, T. Enderle, D.F. Ogletree, D.S. Chemla, P.R. Selvin, S. Weiss, Proc. Natl. Acad. Sci. 93, 6264 (1996); T. Ha, J. Glass, Th. Enderle, D.S. Chemla and S. Weiss, Phys. Rev. Lett. 80, 2093 (1998).

[8] T. Ha, X. Zhuang, H.D. Kim, J.W. Orr, J.R. Williamson and S. Chu, Proc. Natl. Acad. Sci. USA 96, 9077 (1999).

[9] H.D. Kim, G.U. Nienhaus, T. Ha, J.W. Orr, J.R. Williamson and S. Chu, PNAS 99, 4284 (2002).

[10] X. Zhuang, L.E. Bartley, H.P. Babcock, R. Russell, T. Ha, D. Herschlag, and S. Chu, Science 288, 2048 (2000).

[11] R. Russell, X. Zhuang, H.P. Babcock, I.S. Millett, S. Doniach, S. Chu and D. Herschlag, PNAS 99, 155 (2002).

[12] X. Zhuang, T. Ha, H.D. Kim, T. Centner, S. Labeit and S. Chu, PNAS 97, 14241 (2000).

[13] N. G. Walter, K. J. Hampel, K. M. Brown, J. M. Burke, EMBO J. 17, 2378 (1998).

[14] X. Zhuang, H.D. Kim, M. Pereira, H.P. Babcock, N. Walter, and S. Chu, Science 296, 1473-1476 (2002).

[15] Z. Cai and I. Tinoco, Biochemistry 35, 6026 (1996); S. E. Butcher, F. H. Allain, J. Feigon, Nat. Struct. Biol. 6, 212 (1999).

[16] P. B. Rupert, A. R. Ferre-D’Amare, Nature 410, 780 (2001).

[17] The other collaborators are Scott Blanchard, Harold Kim and Ruben Gonzales.

[18] C.G. Kurland, Annu. Rev. Genet. 26, 29 (1992); R. Green and H. F. Noller, Ann. Rev. Biochem. 66, 679 (1997); M. Rodnina, A. Savelsbergh, et al., Nature 385, 37 (1997); T.W. Pape, W. Wintermeyer, et al. EMBO J. 17, 7490 (1998).

[19] N.P. Ban, P. Nissen, et al. Science 289, 905 (2000); Schluenzen, F., A. Tocilj, et al. Cell 102(5), 615 (2000); Wimberly, B. T., D. E. Brodersen, et al. Nature 407, 327 (2000); Yusupov, M. M., G. Z. Yusupova, et al., Science 292, 883 (2001).

[20] The other collaborators are Keith Weninger, Mark Bowen and James Ernst.

[21] See, for example, T.C. Sudhof, Nature 375, 645 (1995); R.C. Lin and R.H. Scheller, Ann. Rev. Cell Dev. Biol. 16, 19 (2000); J. Rizo, T.C. Sudhof, Nat Rev Neurosci. 3(8), 641 (2002).

[22] A.T. Brunger, Curr. Opinion Str. Biol. 11, 163 (2001).

[23] The first unambiguous observation of molecular memory was a single molecule experiment done by H. P. Lu, L. Xun and X. S. Xie, Science 282, 1877 (1998). 